\title{
Natural compounds as inflammation inhibitors
}

\author{
Marc Schumacher - Tom Juncker • Michael Schnekenburger • \\ François Gaascht $\cdot$ Marc Diederich
}

Received: 25 June 2010/ Accepted: 23 August 2010/Published online: 3 May 2011

(C) Springer-Verlag 2011

Since 1998, Dr. Marc Diederich and his research team successfully managed eight cell signaling meetings in Luxembourg attracting more then 5,500 scientists. In January 2010, over 550 scientists and exhibitors came together for the meeting Inflammation 2010, which was held from January 27 th to 30 th. Invited and selected talks followed the keynote session from Dr. Jurg Tschopp (Department of Biochemistry, Switzerland), and more than 210 posters reported recent research results in cell signaling, cancer, inflammation, apoptosis, and innate immunity. This editorial aims at giving insight into natural compounds-related research covered by the meeting.

Known in traditional medicines for several centuries, nature remains a potent source of drugs for the treatment of a large variety of diseases. More than 70 percent of today's anticancer drugs originate from natural compounds or are synthetic derivatives of natural compounds. Highthroughput screening of synthetic libraries gained raising importance during the last 15 years; however, the research on natural products as promising drug candidates is kept actually at a high level.

Recent published data report that innate immunity operates as a complex system to sense and differentiate danger signals from non-danger inputs. Though the immune system will respond to infection or injury, it will remain inactive in case of harmless compounds, food antigens, or microscopes of the gut flora. A deregulated chronic inflammation might lead in some cases to hay

M. Schumacher · T. Juncker · M. Schnekenburger .

F. Gaascht · M. Diederich $(\square)$

Laboratoire de Biologie Moléculaire et Cellulaire du Cancer,

Fondation de Recherche Cancer et Sang, Hôpital Kirchberg,

9 Rue Edward Steichen, 2540 Luxembourg, Luxembourg

e-mail: marc.diederich@lbmcc.lu fever, atherosclerosis, and rheumatoid arthritis. Furthermore, the link between inflammation and cancer, discovered by Virchow in the 19th century, has been confirmed by a large number of groups. In 20 percent of all cases, inflammation is a direct cause of cancer, and in 65 percent of malignancies, the diseases has been caused by obesity, other dietary factors, tobacco smoke, and inhaled pollutants indirectly related to inflammation. The effect of inflammation on human health led to increased interest in research in this area.

Interestingly, the keynote session of the meeting reviewed the importance of inflammasomes as guardians of human body presented by Pr Jurg Tschopp (Department of Biochemistry, Switzerland) of the University of Lausanne. The inflammasome is a complex composed of caspase 1, PYCARD, and NALP; its exact composition depends on the activator involved and is triggered by pathogen-associated molecular patterns (PAMPs), danger-associated molecular patterns (DAMPs), and crystalline substances. Upon activation, ROS-mediated inflammasome complex formation leads to secretion of pro-inflammatory cytokines, such as interleukin-1 $\beta$ (IL-1 $\beta$ ) or IL-18. Inflammasomedependent activity results to pyroptosis, a highly inflammatory form of cell death. Several human diseases are associated with the activation of inflammasomes: autoinflammatory diseases such as cryopyrin-associated periodic syndromes (CAPS), gout. It has been reported recently that high extracellular glucose triggers IL- $1 \beta$ secretion via the NLRP3 inflammasome and the NLRP3-binding protein, TXNIP, and plays a role in triggering type II diabetes. This mentioned research findings of the inflammasome pathway linked to inflammatory diseases might lead to better treatment of the latter, as it is more advantageous to inhibit a deregulated signaling cascade at an upstream position. Clinical trials evaluate the injection of Anakinra (brand 
name Kineret ${ }^{\circledR}$ ), a recombinant form of the naturally occurring antagonist, IL-1RA, for the treatment of gout, CAPS, type II diabetes, and rheumatoid arthritis.

Interestingly, a considerable number of talks reported the implicit use of natural compounds and synthetic derivatives for the treatment of inflammatory diseases. Dr. Ajay Goel (Baylor University Medical Center, USA) raised the attention to the fact that besides genetics, the field of epigenetic gene regulation recently emerged as one of the key driving factors in chronic inflammatory diseases. Goel discussed the regulatory mechanisms that control the expression of various major inflammatory genes and how diet/natural compounds could influence this regulation. Since epigenetic regulatory mechanisms are potentially reversible, this field gains further interest especially related to the re-exploration of anti-inflammatory bioactive food compounds for their properties as potential epigenetic modulators/inhibitors, by targeting the enzymatic activities of chromatin modifier proteins belonging to the DNA methyl transferase (DNMT), histone deacetylase (HDAC), and histone acetyl transferase (HAT) families. To date, a very limited body of experimental evidence addresses molecular mechanisms involved in diet-induced epigenetic alterations in in vitro or pre-clinical studies. Therefore, it is only the beginning of the investigation of epigenetic mechanisms and their modulation by dietary agents in inflammation-related disorders, which provides an exciting opportunity for the development of potential new therapeutic approaches for treatment as well as for prevention of many diseases. In light of this, it is very encouraging to witness the promise of dietary agents as modulators of epigenetic mechanisms. Most epigenetic modulators described belong to the polyphenol family, and for some of those dietary agents, they are already known for other properties; curcumin and epigallocatechin gallate (EGCG) apparently act as HAT inhibitors, DNMT1 inhibitors, as well as miRNA modulators. Genestein, acetyl-keto-beta boswellic acid (AKBA), and caffeic acid were described as DNMT1 inhibitors. Finally, it was mentioned anarcadic acid and garcinol as potent HAT inhibitors, genestein and sulforaphanes as HDAC inhibitors, and resveratrol as a sirtuin (Sirt 1) activator.

NF- $\kappa \mathrm{B}$ (NF-kappaB) is a key transcription factor associated with inflammatory diseases. Pr. Young-Joon Surh (Research Institute of Pharmaceutical Sciences, South Korea) reported the crosstalk between the transcription factor NF- $\kappa \mathrm{B}$ and redox-sensitive transcription factors. In detail, the nuclear factor E2-related factor-2 (Nrf2) activates antioxidant enzymes via binding to antioxidant responsive element (ARE), which strengthens cellular antioxidant potential. Therefore, Nrf2 is a key target gene for inflammatory disorders. Many phytochemicals-such as the organosulfur compound sulforaphane, the polyphenol curcumin and the sesquiterpene zerumboneexerted antioxidant gene expression, by oxidation or covalent modification of thiol groups associated with the presented transcription factors and in this way maintain cellular homeostasis. Though, these mentioned molecules play a crucial role in chemoprevention and cytoprotection.

Nicotine and muscarine, natural products isolated of the tobacco plant and mushrooms, respectively, despite their known toxic to human health, are known to affect the inflammatory pathway. Dr Wounter de Jonge (Academic Medical Center, Amsterdam) reported the effect of especially nicotinic agonists and their stimulation on the vagus nerve, which down-regulate pro-inflammatory cytokine production and modulates JAK/STAT and NF- $\kappa$ B signaling pathways. Future studies have to elucidate the inhibition of the NF- $\kappa \mathrm{B}$ pathway via alpha7 nicotinic acetylcholine receptor $(\alpha 7 \mathrm{nAChR})$ agonists while circumventing their neurotoxicity.

New therapies for the treatment of cystic fibrosis patients are emerging during the last years. Pr. Roberto Gambari (Department of Biochemistry and Molecular Biology, Italy) reported the extraction of two natural products citropten and bergapten from bergamot, which expressed a high inhibition potential against the expression of IL-8; NF- $\kappa$ B plays a key role in IL-8 expression. Furthermore, the investigation into a furocoumarin database led to the discovery of furo(3,2-c)chromen-4-one derivative, which could be a potential pharmaceutical drug to control lung inflammation in CF patients.

Pr. Bharat Aggarwal (Department of Experimental Therapeutics, MD Anderson Cancer Center, Houston, USA) assessed the impact of major risk factors, such as chronic infections, obesity, alcohol, tobacco, radiation, and pollutants, leading to the development of the most common types of cancer. NF- $\kappa$ B and STAT3, two inflammation pathways, are constitutively activated in most cancers and therefore play a crucial role in the development of most cancers. The potential of phytochemicals extracted from traditional Indian and Chinese medicine, like berberine, curcumin and celastrol, for cancer prevention and therapy was discussed.

As one of the most common cancer worldwide with still increasing incident rates, skin cancer requires particular attention and accurate treatments. The signal transduction pathways triggered by the epidermal growth factor receptor (EGFR), protein kinase $\mathrm{C}$ (PKC), the mitogen-activated protein (MAP) kinases c-Jun N-terminal kinase (JNK) $1 / 2$, p38, extracellular-regulated kinase (ERK)1/2, and cyclooxygenase (COX)-2 are activated by exposure of the skin to ultraviolet (UV) A and B irradiation. Inhibition of these signals by natural products should decrease skin carcinogenesis. Pr Zigang Dong and collaborators (Hormel Institute, Austin, MN, USA) were the first to elucidate the effect 
of dietary compounds on specific cell signaling pathways and discovered a series of chemopreventive agents such as EGCG from green tea, myricetin, quercetin, 5-deoxykaempferol, 6-gingerol, and resveratrol, all isolated from fruits and vegetables, which are able to prevent skin carcinogenesis. Fewer secondary effects should result from the natural origin from these drugs in contrast to synthetic therapeutic agents.

Radiotherapy is a pre-operative treatment for patients with a variety of cancer. However, the pathological response level to this therapy is low as pro-survival pathways are activated after radiation by a large percentage of resistant tumor cells. For this reason, Krishnan and coworkers (MD Anderson Cancer Center, Houston, TX, USA) developed a method to sensitize resistant colorectal cancer cells to radiation therapy. As previously reported, the inducible signaling pathway NF- $\kappa \mathrm{B}$ protects cells from cell death via up-regulation of pro-survival genes. The research team used curcumin to increase the efficiency of radiation therapy. Curcumin down-regulated the radiation-induced phosphorylation and degradation of inhibitor of kappa B kinase (IKK). In addition, NF- $\kappa \mathrm{B}$-regulated genes (Bcl-2, Bcl-xL, inhibitor of apoptosis (IAP)-2, COX-2, and cyclin D1) were inhibited. Furthermore, this molecule attenuates in parallel side effects including fatigue associated with radiotherapy. These results led to the identification of curcumin as a radiosensitizer in colorectal cancer. Very recently, the team of Krishnan reported that the histone deacetylase inhibitor vorinostat (suberoylanilide hydroxamic acid, SAHA) exerted a similar sensitization activity compared to curcumin.

A persistent gathering of mature resting $B$ cells in the peripheral blood, bone marrow, and lymphatic tissue is one characteristics of chronic lymphocytic leukemia (CLL). This accumulation is a result of aberrant apoptosis and not due to increased proliferation. Pr. Varsha Gandhi (MD Anderson Cancer Center, Houston, TX, USA) reported about Bcl-2 antagonists, including the polyphenol gossypol and its analog AT-101 derived from the cotton plant, which targets pro-survival proteins. In fact, these compounds displace Bim from Bcl-2's BH3-binding pocket, and then Bim activates BAX, which induces mitochondrial permeabilization and finally leads to cancer cell death.

Flavonoids from plants have been reported to exert antiinflammatory activity in vitro and in vivo. They have an impact on the pro-inflammatory gene expression, like cyclooxygenase-2, nitric oxide synthase and pivotal cytokine. Pr. De-Xing Hou (Department of Biochemical Science and Technology, Japan) reported the effect of flavonoids on protein kinases, which can bind directly to protein kinases or inhibit the phosphorylation of the protein kinases JAK1, Akt, MEK1, Fyn, MKK4, ZAP-70, and $\mathrm{PI} 3 \mathrm{~K}$. It is noteworthy that Akt, PI3K, and ZAP-70 act upstream of the NF- $\kappa \mathrm{B}$ pathway. As protein kinases act on a wide range of intracellular signaling pathways, it is not surprising that flavonoids are reported to exert an impact on multiple biomolecular functions, which will be elucidated in future by the authors.

In vine, resveratrol is produced in large quantity as a response to biotic or abiotic stress; this secondary metabolite activates plant defense mechanisms by antioxidative protection and inhibition of fungi infection. Pr Norbert Latruffe (Laboratory of Biochemistry of Metabolism and Nutrition, INSERM, Dijon, France) investigated the different pathways affected by resveratrol. The latter modulates a large variety of signaling mechanisms, exerts antioxidant potential, and acts as a radical scavenger molecule; this polyphenol inhibits the NF- $\kappa \mathrm{B}$ as well as the COX-2 pathway. The secretion of IL- 6 and IL- 8 is down-regulated, and it blocks cells in the G2/M phase. Recently, the author published a structure-activity relationship study about 14 different resveratrol analogs; it concludes that the trimethoxy derivative of $(Z)$-resveratrol exerts the highest bioactivity.

Marine organisms produce secondary metabolites as an innate defense. Dr. Marc Schumacher (Laboratoire de Biologie Moléculaire et Cellulaire du Cancer, Luxembourg) reported the isolation and bioactivity determination of a variety of natural products isolated from marine sponges and bacteria. The most active compound, the sesterterpene heteronemin, inhibited proteasome activity at nanomolar concentrations. Furthermore, this product induced apoptotic cell death to the resistant cancer cell line K562 and the NF- $\kappa$ B pathway was down-regulated. Future research will be carried out on natural and synthetic derivatives of this terpene.

Rheumatoid arthritis is a severe autoimmune disease resulting in articulation inflammation and bone lesions. With regard to its impressive incident rates, there is a huge need for new, additional therapeutic agents. Dr. Veera R. Konda (KinDex Therapeutics, USA) described a mixture of synthetic substituted cyclopentadiones, named META060. The latter has a US GRAS status as bittering additives in beer. Apart of the NF- $\kappa \mathrm{B}$ pathway inhibition, this mixture blocks the kinases SyK, BTK, GSK3 $\beta$, and PI3K. Bioactivity tests show that this mixture is able to inhibit inflammation in arthritis mice models. These results suggest that these molecules might act as an efficient and safe therapeutics for the treatment of chronic inflammation.

As a conclusion, multiple presentations surveyed the role of inflammation in cancer and other diseases. There exist a large network of different signaling pathways involved in inflammation processes; hence, it is noteworthy more than 10 oral presentations reported the tight link between the NF- $\kappa \mathrm{B}$ pathway and inflammation. So the NF$\kappa \mathrm{B}$ pathway remains one key role player for the development of novel therapeutics for the treatment of diseases based on inflammation. Interestingly, natural compounds 
especially from dietary origins underline the increasing impact of this research area. Hence, the next meeting in Luxembourg-Kirchberg, organized by Dr. Marc Diederich and his research team, will focus on natural compounds as regulators of cell signaling pathways and novel therapeutic tools.
Acknowledgments We would like to thank "Recherches Scientifiques Luxembourg asbl" who organized the meeting. The Fonds National de la Recherche, Luxembourg, Kuwait Petroleum SA, The City of Luxembourg, The Fondation de Recherche "Cancer et Sang", Computer Home and Canon are thanked for additional support. Finally all members of the organization team are thanked for their great organization. 